\title{
GERBES OF CHIRAL DIFFERENTIAL OPERATORS
}

\author{
Vassily Gorbounov, Fyodor Malikov, and Vadim Schechtman
}

1. In this note we compute the cohomological obstruction to the existence of certain sheaves of vertex algebras on smooth varieties. These sheaves have been introduced and studied in the previous work by A. Vaintrob and two of the authors (cf. [MSV] and [MS1]). Hopefully our result clarifies to some extent the constructions of op. cit.

Recall that in [MSV] we discussed two kinds of sheaves on smooth complex algebraic (or analytic) varieties $X$. First, we defined the sheaf of conformal vertex superalgebras $\Omega_{X}^{c h}$, called chiral de Rham algebra. These sheaves are canonically defined for an arbitrary $X$. Second, for some varieties $X$ one can define a purely even counterpart of $\Omega_{X}^{c h}$, a sheaf of graded vertex algebras $\mathcal{O}_{X}^{c h}$, called a chiral structure sheaf, cf. op. cit., $\S 5$. For example, one can define $\mathcal{O}_{X}^{c h}$ for curves, and for flag spaces $G / B$. For an arbitrary $X$, there arises certain cohomological obstruction to the existence of $\mathcal{O}_{X}^{c h}$. The infinitesimal incarnation of this obstruction is calculated in op. cit., $\S 5, \mathrm{~A}$.

In the present note we compute explicitly this obstruction, which turns out to coincide with $2 c h_{2}(X)$, cf. Theorem 3 below. From a different viewpoint, this theorem may be regarded as a geometric interpretation of the second component of Chern character. In nos. 7, 8 we give a generalization of Theorem 3 , cf. Theorem 8.1.

In no. 9 we compute the "conformal anomaly": the obstruction to the existence of a globally defined Virasoro field $L(z)$; it is given by the first Chern class $c_{1}(X) / 2$, cf. Theorem 9.1 .

In particular, Theorem 3 provides a geometric criterion for a manifold to admit a $B U\langle 6\rangle$-structure: those are precisely the manifolds which admit the above mentioned sheaf $\mathcal{O}_{X}^{c h}$ and for which the conformal anomaly vanishes. If such a manifold is Calabi-Yau (i.e., has the trivial canonical bundle) then $\mathcal{O}_{X}^{c h}$ is a sheaf of conformal vertex algebras, cf. Corollary 9.3.

A remark about terminology and notation. As was noted in [MSV], (cf. the last paragraph of $\S 5$ ), the sheaf $\mathcal{O}_{X}^{c h}$ may be regarded as a chiral counterpart of the sheaf $\mathcal{D}_{X}$ of differential operators on $X$. By that reason we prefer in this note to change its notation to $\mathcal{D}_{X}^{c h}$ and call it a sheaf of chiral differential operators on $X$. From this point of view, the sheaf $\Omega_{X}^{c h}$ is a chiral counterpart of the sheaf

Received September 28, 1999.

Fyodor Malikov is partially supported by NSF. 
$\mathcal{D}_{\Omega_{X}}$ of differential operators on the de Rham algebra $\Omega_{X}$ of differential forms, so it would probably deserve the notation $\mathcal{D}_{\Omega_{X}}^{c h}$, cf. [MS2].

For a graded vertex algebra $V, V_{i}$ will denote the component of conformal weight $i$. In this note we shall live in the analytical category. The algebraic case will be treated in a separate publication, cf. [GMS].

We are deeply grateful to A. Beilinson for very enlightening discussions. Our special gratitude goes to him and to H. Esnault for important corrections in the preliminary version of the note. After receiving it, A. Beilinson has sent us very interesting note $[\mathrm{BD}]$ which is closely related to ours.

The work was done while the first and the third author visited the University of Glasgow and Max-Planck-Institut für Matematik; we thank these institutions for the hospitality.

2. Fix an integer $N \geq 1$. Let $O=\mathbb{C}\left[b^{1}, \ldots, b^{N}\right]$ be the polynomial algebra. Following [MSV], define the vertex algebra $D$ as the free bosonic vertex algebra generated by the fields $b^{i}(z)$ of conformal weight 0 and $a^{i}(z)$ of conformal weight $1(i=1, \ldots, N)$, with the OPE

$$
a^{i}(z) b^{j}(w) \sim \frac{\delta_{i j}}{z-w}
$$

the other products being trivial. The algebra $D$ is called the algebra of (algebraic) chiral differential operators on $\mathbb{C}^{N}$.

The conformal weight zero component $D_{0}$ is equal to $\mathbb{C}\left[b_{0}^{1}, \ldots, b_{0}^{N}\right]$, and will be identified with $O$. The component $D_{1}$ is a free $O$-module with the base $b_{-1}^{i}, a_{-1}^{i}$. The submodule $\Omega:=\oplus O \cdot b_{-1}^{i} \subset V_{1}$ will be identified with the $O$-module of 1-differentials $\Omega_{O}^{1}=\oplus O \cdot d b^{i}$, by identifying $b_{-1}^{i}$ with $d b^{i}$. The submodule $T:=\oplus O \cdot a_{-1}^{i}$ will be identified with the $O$-module of vector fields $\oplus O \cdot \partial_{i}$ where $\partial_{i}:=\partial / \partial b^{i}$, by identifying $a_{-1}^{i}$ with $\partial_{i}$.

Let $X$ be an $N$-dimensional smooth complex analytic variety. Let $U \subset X$ be an open subset given together with a coordinate system, i.e., an open embedding $b=\left(b^{1}, \ldots, b^{N}\right): U \longrightarrow \mathbb{C}^{N}$. Let $\mathcal{O}(U)$ denote the algebra of holomorphic functions on $U$. The map $b$ induces an algebra homomorphism $O \longrightarrow \mathcal{O}(U)$. We set $D(U ; b):=\mathcal{O}(U) \otimes_{O} D$. According to [MSV], 6.9, $D(U ; b)$ admits a canonical structure of a conformal vertex algebra. It is called the algebra of (holomorphic) chiral differential operators on $U$, associated with the coordinate system $b$.

If $V \subset U$ is an open subset, the map $b$ induces a coordinate system on $V$, and we get an algebra $D(V ; b)$. When $V$ varies, we get a sheaf of conformal vertex algebras $\mathcal{D}_{(U ; b)}$ on $U$ - the sheaf of holomorphic chiral differential operators on $U$, associated with the coordinate system $b$.

The component $\mathcal{D}_{(U ; b) 0}$ is identified with the sheaf of holomorphic functions $\mathcal{O}_{U}$, and the component $\mathcal{D}_{(U ; b) 1}$ is identified with the direct sum of the sheaf $\Omega_{U}^{1}$ of holomorphic 1-differentials and the sheaf $\mathcal{T}_{U}$ of holomorphic vector fields.

Let us define a two-step filtration on $\mathcal{D}_{(U ; b) 1}$ by

$$
0=F^{2} \mathcal{D}_{(U ; b) 1} \subset \Omega_{U}^{1}=F^{1} \mathcal{D}_{(U ; b) 1} \subset \mathcal{D}_{(U ; b) 1}=F^{0} \mathcal{D}_{(U ; b) 1}
$$


Obviously, $F^{0} / F^{1}=\mathcal{T}_{U}$. The associated graded space

$$
\operatorname{gr}_{F} \mathcal{D}_{(U ; b) 1}=\Omega_{U}^{1} \oplus \mathcal{T}_{U},
$$

does not depend on the choice of a coordinate system $b$, cf. [MSV], 6.2.

Let us define a groupoid $\mathfrak{D}^{c h}(U)$ as follows. The objects of $\mathfrak{D}^{c h}(U)$ are coordinate systems $b$ on $U$. Given two coordinate systems $b,{ }^{\prime} b$, there exists a unique automorphism $g$ of the sheaf $\mathcal{O}_{U}$ taking $b$ to $b^{\prime}$. By definition, the set of morphisms $\operatorname{Hom}_{\mathfrak{D}^{c h}(U)}\left(b,{ }^{\prime} b\right)$ consists of isomorphisms of sheaves of graded vertex algebras $\mathcal{D}_{(U ; b)} \stackrel{\sim}{\longrightarrow} \mathcal{D}_{(U ; ' b)}$ which induce $g$ on the conformal weight zero components.

This groupoid will be called the groupoid of (holomorphic) chiral differential operators on $U$. When $U$ varies, we get a stack (champ) of groupoids, i.e., a gerbe $\mathfrak{D}_{X}^{c h}$ on $X$, called the gerbe of (holomorphic) chiral differential operators on $X$.

Concerning the J.Giraud's language of gerbes, see [G].

Recall that the set of equivalence classes of gerbes on $X$ bounded by a given abelian sheaf $\mathcal{F}$ is identified with the group $H^{2}(X ; \mathcal{F})$. Namely, if $\mathfrak{G}$ is such a gerbe, choose an open covering $\mathcal{U}=\left\{U_{i}\right\}$ of $X$ such that for each $U_{i}$ the groupoid $\mathfrak{G}(U)$ is nonempty and connected. Choose objects $\mathcal{G}_{i} \in \mathfrak{G}\left(U_{i}\right)$. On the pairwise intersections there exist isomorphisms $\phi_{i j}:\left.\left.\mathcal{G}_{i}\right|_{U_{i j}} \stackrel{\sim}{\longrightarrow} \mathcal{G}_{j}\right|_{U_{i j}}$. The "discrepancies" $c_{i j k}:=\phi_{i j} \phi_{j k} \phi_{i k}^{-1}$ (on triple intersections) define a Cech 2-cocycle in $Z^{2}(\mathcal{U} ; \mathcal{F})$ which represents the class $c(\mathfrak{G})$.

Let $E$ be a vector bundle over $X$ given by a Cech 1-cocycle $g=\left\{g_{i j}\right\} \in$ $Z^{1}\left(\mathcal{U} ; G L_{n}\left(\mathcal{O}_{X}\right)\right)$. Let $\Omega_{X}^{[i}$ denote the truncated and shifted de Rham complex

$$
\Omega_{X}^{[i}=\left(\Omega_{X}^{i} \longrightarrow \Omega_{X}^{i+1} \longrightarrow \cdots\right)
$$

so that $\Omega_{X}^{i}$ leaves in degree 0 . Let us define a 2-cocycle $c h_{2}^{(g)}(E) \in Z^{2}\left(\mathcal{U} ; \Omega_{X}^{[2}\right)$ with components

$$
\left.\operatorname{ch}_{2}^{(g)}(E)=\left(\frac{1}{2} \operatorname{tr}\left(\operatorname{dlog} g_{i j} \wedge \operatorname{dlog} g_{j k}\right), \frac{1}{6} \operatorname{tr}\left(\operatorname{dlog} g_{i j}\right)^{3}\right)\right) .
$$

One can check that the cohomology class $\operatorname{ch}_{2}(E) \in H^{2}\left(X ; \Omega_{X}^{[2}\right)$ of the cocycle(2.5) does not depend on the choice of trivialization.

Let $\Omega_{X}^{i, c l} \subset \Omega_{X}^{i}$ denote the subsheaf of closed forms. Since we leave in the analytical situation, an obvious map $\Omega_{X}^{i, c l} \longrightarrow \Omega_{X}^{[i}$ is a quasiisomorphism, so that we can consider $c h_{2}(E)$ as an element of $H^{2}\left(X ; \Omega_{X}^{2, c l}\right)$.

Assume that variety $X$ and vector bundle $E$ is algebraic. Recall that we have the Chern classes $c_{i}^{(\mathcal{K})}(E) \in H^{i}\left(X ; \mathcal{K}_{i, X}\right)$ with values in the Zariski sheaves $\mathcal{K}_{i, X}$ of Quillen $K$-groups (cf. for example [S]). If

$$
c_{i}(E) \in H^{i}\left(X ; \Omega_{X}^{i, c l}\right),
$$


denote the image of these classes under the natural map induced by the "dlog" $\operatorname{map} \mathcal{K}_{i, X} \longrightarrow \Omega_{X}^{i, c l}$ then

$$
\operatorname{ch}_{2}(E)=\frac{1}{2} c_{1}(E)^{2}-c_{2}(E)
$$

Yet another way of defining these classes is as follows. Consider the Zariski cohomology groups $H_{Z \text { Zar }}^{i}\left(X ; \Omega_{X}^{i i}\right)$. These groups satisfy the standard properties of a cohomology theory needed to define the theory of Chern classes $c_{i}^{\text {(Zar) }}(E) \in$ $H_{\text {Zar }}^{i}\left(X ; \Omega_{X}^{[i}\right)$, cf. [Gr] (this fact was communicated to us by A. Beilinson). The classes $c_{i}(E)$ coincide with the image of $c_{i}^{(\mathrm{Zar})}(E)$ under the natural map from Zariski to the analytical cohomology.

Note that the image of $\operatorname{ch}_{2}(E)$ in $H^{2}\left(X ; \Omega_{X}^{2}\right)$ is equal to

$$
\operatorname{ch}_{2}^{\text {Hodge }}(E):=\frac{1}{2} c_{1}^{\text {Hodge }}(E)^{2}-c_{2}^{\text {Hodge }}(E),
$$

where $c_{i}^{\text {Hodge }}(E) \in H^{i}\left(X ; \Omega_{X}^{i}\right)$ are the Chern classes "style Hodge", [Gr].

Now we can formulate our main result.

3. Theorem. The lien of the gerbe $\mathfrak{D}_{X}^{c h}$ is canonically isomorphic to the sheaf of closed holomorphic 2-differentials $\Omega_{X}^{2, c l}$.

The equivalence class $c\left(\mathfrak{D}_{X}^{c h}\right) \in H^{2}\left(X ; \Omega_{X}^{2, c l}\right)$ is equal to

$$
c\left(\mathfrak{D}_{X}^{c h}\right)=2 c h_{2}\left(\mathcal{T}_{X}\right)
$$

Here $\mathcal{T}_{X}$ denotes the tangent bundle.

This theorem means that a "sheaf of chiral differential operators" is not a uniquely defined object associated with $X$, but an object of a canonically defined groupoid $\Gamma\left(X ; \mathfrak{D}_{X}^{c h}\right)$. This groupoid is empty if the class $(3.1)$ is non-trivial.

If (3.1) is trivial then this groupoid is equivalent to the groupoid of $\Omega_{X}^{2, c l}$ torseurs on $X$. Thus, in this case the set of isomorphism classes of $\Gamma\left(X ; \mathfrak{D}_{X}^{c h}\right)$ is isomorphic to $H^{1}\left(X ; \Omega_{X}^{2, c l}\right)$, and the automorphism group of an object is isomorphic to $H^{0}\left(X ; \Omega_{X}^{2, c l}\right)$.

From this one sees immediately that for curves the obstruction vanishes, and the above groupoid is trivial: one can define canonically "the" sheaf of chiral differential operators $\mathcal{D}_{X}^{c h}$. For the explicit construction, see (6.2) below.

The same is true for the flag spaces $G / B$. This explains the constructions of [MSV]. As a negative example, note that for the projective spaces of dimension greater than 1, the class (3.1) is non-zero.

Below we shall not prove (3.1) as stated but check a slightly weaker statement:

3.1. Claim. The image of $c\left(\mathfrak{D}_{X}^{c h}\right)$ in $H^{2}\left(X ; \Omega_{X}^{2}\right)$ is equal to $2 \operatorname{ch}_{2}^{\text {Hodge }}\left(\mathcal{T}_{X}\right)$.

For the complete proof of (3.1), see [GMS]. 
4. Let us explain a local statement which implies Theorem 3. Let $\widehat{O} \supset O$ be the algebra of the Taylor power series in variables $b^{i}$ convergent in some neighbourhood of the origin. Let $G$ be the group whose elements are $N$-tuples $g=\left(g^{1}(b), \ldots, g^{N}(b)\right)$ where $g^{i} \in \widehat{O}$ and the Jacobian matrix $\partial g:=\left(\partial_{i} g^{j}\right)$ is non-degenerate. The composition is defined by

$$
\left(g_{1} g_{2}\right)^{i}(b)=g_{2}^{i}\left(g_{1}^{1}(b), \ldots, g_{1}^{N}(b)\right) .
$$

The group $G$ acts in the obvious way from the right on the algebra $\widehat{O}$.

We have

$$
\partial\left(g_{1} g_{2}\right)=\partial g_{1} \partial g_{2 g_{1}}
$$

Here we use the notation

$$
h_{g}(b):=h(g(b))
$$

We will denote by $\Omega_{\widehat{O}}^{1}$ (resp., $\mathcal{T}_{\widehat{O}}$ ) the module of germs of holomorphic 1differentials (resp., holomorphic vector fields). It is a free $\widehat{O}$-module with the base $\left\{d b^{i}\right\}$ (resp., $\left\{\partial_{i}\right\}$ ).

Let us consider the vertex algebra $\widehat{D}:=\widehat{O} \otimes_{O} D$. We have $\widehat{D}_{0}=O$, and $\widehat{D}_{1}$ has a canonical two step filtration defined as in (2.2), with $F^{1} \widehat{D}_{1}=\Omega_{\widehat{O}}^{1}, \widehat{D}_{1} / F^{1} \widehat{D}_{1}=$ $\mathcal{T}_{\widehat{O}}$.

Let us call an automorphism of $\widehat{D}$ natural if it induces an automorphism $g \in G$ on $\widehat{D}_{0}$, and the induced by $g$ automorphism on $g r_{F} \widehat{D}_{1}$.

We want to describe the group $G^{\sim}$ of all natural automorphisms of $\widehat{D}$. In coordinates, a natural automorphism is given by a pair $(g, h)$ where $g \in G$ and $h \in M_{N}(\widehat{O})$ is an $N \times N$-matrix over $\widehat{O}$. By definition, such a pair acts on the generating fields as follows

$$
\begin{gathered}
\left(b^{i} \circ(g, h)\right)(z)=g^{i}(b)(z), \\
\left(a^{i} \circ(g, h)\right)(z)=a^{j} \phi^{j i}(g)(z)+b^{k}(z)^{\prime} h^{k i}(z),
\end{gathered}
$$

(this will be the right action). Here the matrix $\phi(g)=\left(\phi^{i j}(g)\right)$ is defined by

$$
\phi(g)=(\partial g)^{-1 t}
$$

where $\left(a^{t}\right)^{i j}=a^{j i}$.

The straightforward computation of OPE shows that a pair $(g, h)$ defines an automorphism of the vertex algebra $\widehat{D}$ if and only if the matrix $h$ satisfies the equations

$$
\begin{gathered}
h^{t} \phi(g)+\phi^{t}(g) h=\psi(g), \quad \text { and } \\
\phi^{p i} \partial_{p} h^{s j}-\phi^{p j} \partial_{p} h^{s i}+\partial_{s} \phi^{p i} h^{p j}+\partial_{s} h^{p i} \phi^{p j}-\partial_{s} \partial_{r} \phi^{p i} \partial_{p} \phi^{r j}=0,
\end{gathered}
$$


where $\psi(g)=\left(\psi^{i j}(g)\right)$ is a symmetric matrix

$$
\psi^{i j}(g)=\partial_{r} \phi^{p i}(g) \partial_{p} \phi^{r j}(g)
$$

(we imply everywhere the summation over the repeating indices).

The computation similar to that in the proof of Theorem 3.8 from [MSV], shows that the composition on $G^{\sim}$ is given by the formula

$$
\left(g_{1}, h_{1}\right)\left(g_{2}, h_{1}\right)=\left(g_{1} g_{2}, h_{1} \phi\left(g_{2}\right)_{g_{1}}+\partial g_{1} h_{2 g_{1}}+\alpha\left(g_{1}, g_{2}\right)\right),
$$

where $\alpha\left(g_{1}, g_{2}\right)=\left(\alpha^{i j}\left(g_{1}, g_{2}\right)\right)$ is given by

$$
\alpha^{i j}\left(g_{1}, g_{2}\right)=\partial_{i} \phi^{p q}\left(g_{1}\right) \partial_{p}\left[\phi^{q j}\left(g_{2}\right)_{g_{1}}\right] .
$$

This describes the group $G^{\sim}$. The unit is $(1,0)$. Let us introduce a bigger group $G^{\prime}$ consisting of all pairs $(g, h)$ as above, with the multiplication (4.8), but with $h$ not necessarily satisfying the conditions $(4.6 \mathrm{a}, \mathrm{b})$. One has to check the associativity of multiplication, which is straightforward. Obviously, $G^{\sim} \subset G^{\prime}$.

It is convenient to introduce in $G^{\prime}$ the new coordinates by setting

$$
(g, h)^{\sim}:=(g, 0)(1, h)=\left(g, h \partial g^{-1 t}\right) .
$$

In new coordinates the multiplication in $G^{\prime}$ looks as follows

$$
\left(g_{1}, h_{1}\right)^{\sim}\left(g_{2}, h_{2}\right)^{\sim}=\left(g_{1} g_{2}, h_{1}+\partial g_{1} h_{2 g_{1}} \partial g_{1}^{t}+\beta\left(g_{1}, g_{2}\right)\right)^{\sim},
$$

where

$$
\beta\left(g_{1}, g_{2}\right)=\alpha\left(g_{1}, g_{2}\right) \partial\left(g_{1} g_{2}\right)^{t}
$$

In particular,

$$
(1, h)(g, 0)=\left(h, \partial g \cdot h_{g} \cdot \partial g^{t}\right) .
$$

We have an obvious epimorphism $G^{\prime} \longrightarrow G,(g, h) \mapsto g$, and the formula (4.12) shows that its kernel, as a $G$-module, may be identified with the $G$-module of quadratic differentials $\Omega_{\widehat{O}}^{1 \otimes 2}:=\Omega_{\widehat{O}}^{1} \otimes_{\widehat{O}} \Omega_{\widehat{O}}^{1}$, i.e., we have the group extension

$$
0 \longrightarrow \Omega_{\widehat{O}}^{1 \otimes 2} \longrightarrow G^{\prime} \longrightarrow G \longrightarrow 1 \text {. }
$$

Equation (4.10) means that if we chose a section of the epimorphism $G^{\prime} \longrightarrow G$ as $g \mapsto(g, 0)$ then the extension (4.13) corresponds to the 2-cocycle $\beta\left(g_{1}, g_{2}\right) \in$ $Z^{2}\left(G ; \Omega_{\widehat{O}}^{2}\right)$. The equation of a cocycle (equivalent to the associativity of multiplication in $G^{\prime}$ ) reads as

$$
\beta\left(g_{1}, g_{2}\right)-\beta\left(g_{1}, g_{2} g_{3}\right)+\beta\left(g_{1} g_{2}, g_{3}\right)-g_{1}^{*} \beta\left(g_{2}, g_{3}\right)=0 .
$$

A little computation using the formula $\partial_{i}\left(A^{-1}\right)=-A^{-1} \partial_{i} A \cdot A^{-1}$ gives the following expression for $\beta$ :

$$
\beta^{i j}\left(g_{1}, g_{2}\right)=\partial\left(g_{1}^{-1}\right)^{q a} \partial_{i} \partial_{a} g_{1}^{b} \partial_{j}\left[\left(\partial_{b} g_{2}^{r}\right)_{g_{1}}\right]\left(\partial g_{2}^{-1}\right)_{g_{1}}^{r q},
$$

which may be rewritten in the following compact form:

$$
\beta\left(g_{1}, g_{2}\right)=\operatorname{tr}\left\{\partial g_{1}^{-1} d \partial g_{1} \otimes g_{1}^{*}\left(d \partial g_{2} \cdot \partial g_{2}^{-1}\right)\right\} .
$$


5. Now let us take care of the subgroup $G^{\prime \prime} \subset G^{\prime}$ given inside $G^{\prime}$ by the equation (4.6a). Since the matrix $\psi(g)$ is symmetric, and the matrix $h$ is arbitrary, the above equation has $N(N-1) / 2$-dimensional (over $\widehat{O}$ ) space of solutions.

For example, for $N=1,(4.6)$ has a unique solution, and $G^{\prime \prime}=G$, namely

$$
h(g)=g^{\prime \prime 2} / 2 g^{\prime 3} .
$$

Now let us treat the case of an arbitrary $N$. First of all, $\psi(1)=0$ and $\phi(1)=1$, and the equation (4.6) means simply that the matrix $h$ is skew symmetric. This means that $G^{\prime \prime}$ is included in a group extension

$$
0 \longrightarrow \Omega_{\widehat{O}}^{2} \longrightarrow G^{\prime \prime} \longrightarrow G \longrightarrow 1
$$

It is easy to write down a particular solution of (4.6a), namely

$$
h(g)=\phi^{t}(g)^{-1} \psi(g) / 2 .
$$

This gives a section of (5.2), given by

$$
g \mapsto(g, h(g))=:(g, s(g))^{\sim} .
$$

If $c\left(g_{1}, g_{2}\right) \in Z^{2}\left(G ; \Omega_{\widehat{O}}^{2}\right)$ is the cocycle corresponding to this choice of the section then we have

$$
c\left(g_{1}, g_{2}\right)=\beta\left(g_{1}, g_{2}\right)+s\left(g_{1}\right)-s\left(g_{1} g_{2}\right)+\partial g_{1} s\left(g_{2}\right)_{g_{1}} \partial g_{1}^{t} .
$$

In other words, $c$ is cohomologous to $\beta$. But as far as we know that the cohomology class of $\beta$ comes from a cohomology class from $H^{2}\left(G ; \Omega_{\hat{O}}^{2}\right)$, this last class is unique and may be given simply by the skew symmetrization of $\beta$, i.e., by

$$
\begin{aligned}
\gamma\left(g_{1}, g_{2}\right)=\operatorname{tr}\left\{\partial g_{1}^{-1} d \partial g_{1}\right. & \left.\wedge g_{1}^{*}\left(d \partial g_{2} \cdot \partial g_{2}^{-1}\right)\right\}= \\
& \operatorname{tr}\left\{d \partial g_{1} \cdot \partial g_{1}^{-1} \wedge \partial g_{1} \cdot g_{1}^{*}\left[d \partial g_{2} \cdot \partial g_{2}^{-1}\right] \cdot \partial g_{1}^{-1}\right\}
\end{aligned}
$$

Comparing the expression (5.6) with the formula (4.5) from [TT] we see that the cocycle $\gamma$ is nothing but the universal cocycle of $G$ representing the characteristic class corresponding to the invariant polynomial $\operatorname{tr}(A B)$ (on pairs of $N \times N$ matrices), i.e., it represents the component of the Chern character $c_{1}^{2}-2 c_{2}$.

Thus, we arrive at

5.1. Proposition. The group $G^{\prime \prime}$ is included in the group extension

$$
0 \longrightarrow \Omega_{\widehat{O}}^{2} \longrightarrow G^{\prime \prime} \longrightarrow G \longrightarrow 1 \text {. }
$$

The cohomology class of this extension is equal to $2 \mathrm{ch}_{2}^{\text {Hodge }}:=\left(c_{1}^{\text {Hodge }}\right)^{2}-$ $2 c_{2}^{\text {Hodge }} \in H^{2}\left(G ; \Omega_{\widehat{O}}^{2}\right)$ where $c_{i}^{\text {Hodge }} \in H^{i}\left(G ; \Omega_{\widehat{O}}^{i}\right)$ are the universal Chern classes "style Hodge". 
6. Finally, group $G^{\sim}$ is given inside $G^{\prime \prime}$ by the equation (4.6b). For $g=1$ this equation simply means that the form $h^{i j} d b^{i} \wedge d b^{j}$ is closed (we are grateful to A. Beilinson for this remark).

Therefore, the kernel of the obvious map $G^{\sim} \longrightarrow G$ is isomorphic to the $G$-module $\Omega_{\widehat{O}}^{2, c l}$ of closed 2-forms.

6.1. Lemma. The map $G^{\sim} \longrightarrow G$ is surjective.

Proof. Note that in $[\mathrm{MSV}], \S 5$ one has lifted an arbitrary element of the Lie algebra $W_{N}:=\operatorname{Lie}(G)$ to a derivation $\pi(\tau)$ of $\widehat{D}$. Since every $g \in G$ is the exponent of some vector field $\tau$, we can take $\tilde{g}=\exp (\pi(\tau))$ as a lifting of $g$.

The above considerations, together with Proposition 5.1, imply

6.2. Theorem. The group $G^{\sim}$ of all natural automorphisms of the vertex algebra $\widehat{D}$ is included in the group extension

$$
0 \longrightarrow \Omega^{2, c l} \longrightarrow G^{\sim} \longrightarrow G \longrightarrow 1
$$

The image of the cohomology class of this extension in $H^{2}\left(G ; \Omega_{\widehat{O}}^{2}\right)$ is equal to $2 c h_{2}^{\text {Hodge }}$.

Consider the case $N=1$. In this case $G^{\sim}=G^{\prime \prime}=G$. Thus, every automorphism $g \in G$ lifts uniquely to a natural automorphism of $\widehat{D}$ given by

$$
\tilde{g}=\left(g, g^{\prime \prime 2} / 2 g^{\prime 3}\right)
$$

cf. (5.1). This corresponds to the fact that for curves the sheaf $\mathcal{D}_{X}^{c h}$ is uniquely defined. Note that the formula [MSV], (5.23b) is a particular case of (6.2) (for $\left.g(b)=b^{-1}\right)$.

Our main Theorem 3 (with (3.1) replaced by a weaker Claim 3.1) is an immediate consequence of Theorem 6.2.

7. Let $E$ be a vector bundle over $X, \Lambda E$ its exterior algebra. Proceeding in a similar way as above, we can produce a gerbe $\mathfrak{D}_{\Lambda E}^{c h}$ of chiral differential operators on $\Lambda E$, also bounded by the lien $\Omega_{X}^{2, c l}$.

Let us explain how to do this. Let us describe the local model. In the notations of no. 2 , fix an integer $M \geq 0$. Let us define a vertex superalgebra $D_{\Lambda} \supset D$ which is generated by the free bosonic fields $b^{i}(z), a^{i}(z)$ as in loc. cit. and free odd fields $\phi^{j}(z)$ of conformal weight 0 and $\psi^{j}(z)$ of conformal weight $1(j=1, \ldots, M)$, with the OPE $(2.1)$ and

$$
\psi^{i}(z) \phi^{j}(w) \sim \frac{\delta_{i j}}{z-w},
$$

the other products being trivial. Set $\widehat{D}_{\Lambda}:=\widehat{O} \otimes_{O} D_{\Lambda}$. Consider the group $H$ which is the semidirect product of the group of coordinate changes $G$ from 
no. 4, the group of $M \times M$ matrices $\operatorname{Mat}_{M}(\widehat{O})$, with the obvious action of $G$ on $\operatorname{Mat}_{M}(\widehat{O})$. The group $H$ will be the analogue of the group $G$ in our situation.

Let us call an automorphism of the superalgebra $\widehat{D}_{\Lambda}$ admissible if it has the form

$$
\begin{gathered}
\tilde{b}^{i}(z)=g^{i}(b)(z) \\
\tilde{a}^{i}(z)=a^{j} c^{j i}(g)(z)-\psi^{p} \phi^{q} A^{q s} c^{k i} \partial_{k} A^{-1 s p}(z)+b^{j}(z)^{\prime} h^{i j}(z), \\
\tilde{\phi}^{i}(z)=\phi^{j} A^{j i}(z) \\
\tilde{\psi}^{i}(z)=\psi^{j} A^{-1 i j}(z)
\end{gathered}
$$

for some $(g, A) \in H$ and $h \in \operatorname{Mat}_{N}(\widehat{O})$. Here $c(g):=(\partial g)^{-1 t}$ (we have changed the notation from (4.5), since we already have $\phi$ 's).

We want to compute the group $\tilde{H}$ of all admissible automorphisms of $\widehat{D}_{\Lambda}$. The composition will be

$$
\begin{aligned}
& \left(g_{1}, A_{1}, h_{1}\right)\left(g_{2}, A_{2}, h_{2}\right)= \\
& \quad\left(g_{1} g_{2}, A_{1} A_{2 g_{1}}, h_{1} c\left(g_{2}\right)_{g_{1}}+\partial g_{1} h_{2 g_{1}}+\alpha\left(g_{1}, g_{2}\right)-\gamma\left(g_{1}, g_{2}\right)\right)
\end{aligned}
$$

where $\alpha\left(g_{1}, g_{2}\right)$ is given by (4.9), and

$$
\gamma\left(g_{1}, A_{1} ; g_{2}, A_{2}\right)^{i j}=\partial_{i} A_{1}^{-1 p r} A_{1}^{r q} A_{2 g_{1}}^{q s} c\left(g_{2}\right)_{g_{1}}^{k j} \partial_{k} A_{2 g_{1}}^{-1 s p} .
$$

Set

$$
\delta\left(g_{1}, A_{1} ;, g_{2}, A_{2}\right)=\gamma\left(g_{1}, A_{1}, g_{2}, A_{2}\right) \partial\left(g_{1} g_{2}\right)^{t},
$$

cf. (4.11). A little computation gives

7.1. Lemma. We have

$$
\delta\left(g_{1}, A_{1}, g_{2}, A_{2}\right)=\operatorname{tr}\left\{d A_{1} \cdot A_{1}^{-1} \otimes A_{1} g_{1}^{*}\left(d A_{2} \cdot A_{2}^{-1}\right) A_{1}^{-1}\right\} .
$$

Cf. (4.16).

Let $H^{\prime}$ be the group of all triples $(g, A, h)$ as above, with the composition given by (7.3). We arrive at

7.2. Proposition. The group $H^{\prime}$ is included in the extension

$$
0 \longrightarrow \Omega_{\widehat{O}}^{1 \otimes 2} \longrightarrow H^{\prime} \longrightarrow H \longrightarrow 1
$$

given by the two-cocycle

$$
\epsilon\left(g_{1}, A_{1} ; g_{2}, A_{2}\right)=\beta\left(g_{1}, g_{2}\right)-\gamma\left(g_{1}, A_{1} ; g_{2}, A_{2}\right),
$$

where $\beta$ (resp. $\gamma$ ) is given by (4.16) (resp. by (7.6)). 
8. Returning to the global situation of the beginning of the preceeding subsection (we assume that $\operatorname{dim}(X)=N, \operatorname{rk}(E)=M$ ), we define for a sufficiently small $U \subset X$ the groupoid $\mathfrak{D}_{\Lambda E}^{c h}(U)$ whose objects are pairs

(a coordinate system on $U$, a trivialization of $E$ over $U$ ),

and morphisms are defined as in no. 2, using the admissible isomorphisms of the corresponding vertex superalgebras. This way we get the gerbe $\mathfrak{D}_{\Lambda E}^{c h}$ over $X$.

8.1. Theorem. The gerbe $\mathfrak{D}_{\Lambda E}^{c h}$ is bounded by the lien $\Omega_{X}^{2, c l}$. Its equivalence class is equal to

$$
c\left(\mathfrak{D}_{\Lambda E}^{c h}\right)=2 c h_{2}\left(\mathcal{T}_{X}\right)-2 c h_{2}(E) .
$$

The proof goes along the same lines as in 5, 6, starting from Proposition 7.2. Again this way we get a slightly weaker statement than (8.1), namely that the image of $c\left(\mathfrak{D}_{\Lambda E}^{c h}\right)$ in $H^{2}\left(X ; \Omega_{X}^{2}\right)$ is equal to $2 c h_{2}^{\text {Hodge }}\left(\mathcal{T}_{X}\right)-2 c h_{2}^{\text {Hodge }}(E)$, referring for the complete proof of (8.1) to [GMS].

If $E=\mathcal{T}_{X}$ or $\Omega_{X}^{1}$ then the obstruction (8.1) vanishes. If $E=\Omega_{X}^{1}$ then the gerbe $\mathfrak{D}_{\Lambda E}^{c h}$ admits a canonical global section - the chiral de Rham complex constructed in [MSV].

9. Let us return to the situation of nos. 4-6. Consider an automorphism of $\widehat{D}$ given by an element $\tilde{g}=(g, h(g))$ where $h(g)$ is given by (5.3). Recall that $\widehat{D}$ admits a canonical structure of a conformal vertex algebra, with the Virasoro field

$$
L(z)=a^{i}(z) b^{i}(z)^{\prime}
$$

9.1. Theorem. Under the automorphism $\tilde{g}$, the Virasoro field $L(z)$ is transformed as follows

$$
L(z)^{\sim}=L(z)-\frac{1}{2}(\operatorname{Tr} \log \partial g(b(z)))^{\prime \prime} .
$$

Here the tilde denotes the result of transformation under $\tilde{g}$.

Compare this formula with [MSV], Theorem 4.2.

Proof. Let us consider the one-dimensional case for simplicity. We have, in the obvious notations,

$$
L^{\sim}=a(z)_{-1}^{\sim} b(z)_{-1}^{\sim} \mathbf{1}=g(b(z))_{-1}\left[a_{-1} g_{b}^{\prime}\left(b_{0}\right)^{-1}+b_{-1} \frac{g_{b b}^{\prime \prime}\left(b_{0}\right)^{2}}{2 g_{b}^{\prime}\left(b_{0}\right)^{3}}\right] \mathbf{1} .
$$

We have the Taylor formula

$$
g(b(z))=g\left(b_{0}\right)+g_{b}^{\prime}\left(b_{0}\right) \Delta b(z)+\frac{1}{2} g_{b b}^{\prime \prime}\left(b_{0}\right) \Delta b(z)^{2}+\frac{1}{6} g_{b b b}^{\prime \prime \prime}\left(b_{0}\right) \Delta b(z)^{3}+\cdots
$$


where

$$
\Delta b(z)=b(z)-b_{0}=b_{-1} z+b_{1} z^{-1}+b_{-2} z+b_{2} z^{-2}+\cdots .
$$

The component $g(b(z))_{-1}$ is the coefficient at $z$ of the expression (9.4). This is of course the infinite sum, but we are interested only in the terms which give the nonzero contribution after plugging into (9.3). These are the terms containing (except for $b_{0}$ ) the monomials $b_{-1}, b_{-1}^{2} b_{1}$ and $b_{-2} b_{1}$ (the last two would interact with $a_{-1}$ in (9.3)). Let us write down these terms:

$$
g(b(z))_{-1}=g_{b}^{\prime}\left(b_{0}\right) b_{-1}+g_{b b}^{\prime \prime}\left(b_{0}\right) b_{-2} b_{1}+\frac{1}{2} g_{b b b}^{\prime \prime \prime}\left(b_{0}\right) b_{-1}^{2} b_{1}+\cdots .
$$

Substituting this into (9.3), we get

$$
\begin{aligned}
& L^{\sim}= \\
& L-\frac{1}{2}\left[\left(2 g_{b b}^{\prime \prime}\left(b_{0}\right) g_{b}^{\prime}\left(b_{0}\right) b_{-2}+g_{b b b}^{\prime \prime \prime}\left(b_{0}\right) g_{b}^{\prime}\left(b_{0}\right) b_{-1}^{2}-g_{b b}^{\prime \prime}\left(b_{0}\right)^{2} b_{-1}^{2}\right) g_{b}^{\prime}\left(b_{0}\right)^{-2}\right] \mathbf{1} .
\end{aligned}
$$

Let $L_{-1}: \widehat{D} \longrightarrow \widehat{D}$ denote the canonical derivation of our vertex algebra. It remains to notice that the anomalious term in (9.5) is equal to

$$
-\frac{1}{2} L_{-1}\left\{g_{b b}^{\prime \prime}\left(b_{0}\right) g_{b}^{\prime}\left(b_{0}\right)^{-1} b_{-1} \mathbf{1}\right\}
$$

This follows from the Leibniz rule and the formulas $L_{-1}\left(b_{0} \mathbf{1}\right)=b_{-1} \mathbf{1}$; $L_{-1}\left(b_{-1} \mathbf{1}\right)=2 b_{-2} \mathbf{1}$. Therefore, the corresponding field is

$$
-\frac{1}{2}\left\{g_{b b}^{\prime \prime}(b(z)) b(z)^{\prime} g_{b}^{\prime}(b(z))^{-1}\right\}^{\prime}=-\frac{1}{2}\left\{\log g_{b}^{\prime}(b(z))\right\}^{\prime \prime} .
$$

This proves the theorem in the one-dimensional case.

The higher dimensional case is checked in a similar manner. We leave it to the reader.

9.2. Note that (9.2) implies that the Fourier components $L_{0}, L_{-1}$ are always conserved as it should be: our sheaves $\mathcal{D}_{X}^{c h}$ are always the sheaves of graded vertex algebras.

9.3. Corollary. Let $X$ be smooth variety with the trivial canonical bundle and with $c_{2}\left(\mathcal{T}_{X}\right)=0$. Then each sheaf $\mathcal{D}_{X}^{c h} \in \mathfrak{D}_{X}^{c h}(X)$ admits a structure of a sheaf of conformal vertex algebras.

Indeed, since the canonical bundle is trivial, we can choose a coordinate atlas of $X$ such that the Jacobians of the transition functions are constants. For such an atlas, the anomalious terms in (9.2) disappear. If $c_{2}\left(\mathcal{T}_{X}\right)=0$ then we glue our sheaves $\mathcal{D}_{X}^{c h}$ using the gluing automorphisms (4.4) were the matrices $h$ can be written as $h=h_{1}+h_{2}$ where $h_{1}$ is given by (5.3) and $h_{2}$ is a skew symmetric matrix. Note that a correction by a skew symmetric matrix does not change the Virasoro field: we have

$$
b^{i}(z)^{\prime}\left[a^{i}(z)+b^{k}(z)^{\prime} h^{k i}(z)\right]=b^{i}(z)^{\prime} a^{i}(z)+b^{i}(z)^{\prime} b^{k}(z)^{\prime} h^{k i}(z)=b^{i}(z)^{\prime} a^{i}(z),
$$

if $\left(h^{k i}\right)$ is skew symmetric. Therefore, the Virasoro field is conserved in our sheaf. 
9.4. Similarly to nos. 7,8 , we may add the exterior bundle to the picture. The corresponding conformal anomaly will be equal to

$$
\frac{1}{2}\left(c_{1}\left(\mathcal{T}_{X}\right)-c_{1}(E)\right)
$$

We leave the details to the reader.

\section{References}

[BD] A. Beilinson and V. Drinfeld, Excerpt from "Chiral Algebras II" on chiral tdo and Tate structures, preprint, 1999.

[G] J.Giraud, Cohomologie non abélienne, Die Grundlehren der mathematischen Wissenschaften, Band 179., Springer-Verlag, Berlin-New-York, 1971.

[GMS] V. Gorbounov, F. Malikov, and V. Schechtman, Chiral algebras of differential operators, in preparation.

[Gr] A. Grothendieck, Classes de Chern et répresentations linéaires des groupes discrets, Dix exposés sur la cohomologie des schémas, Masson \& Cie, Paris-North HollandAmsterdam, 1968, pp. 215-305.

[MS1] F. Malikov and V. Schechtman, Chiral de Rham complex. II, D.B. Fuchs' 60-th Anniversary volume (1999), math.AG/9901065.

[MS2] _ Chiral Poincaré duality, Math. Res. Lett. 6 (1999), 533-546; math. AG/9905008.

[MSV] F. Malikov, V. Schechtman, and A. Vaintrob, Chiral de Rham complex, Comm. Math. Phys. 204 (1999), 439-473.

[S] V. Schechtman, Riemann-Roch theorem and the Atiyah-Hirzebruch spectral sequence, Usp. Mat. Nauk 35 (1980), 179-180. (Russian)

[TT] D. Toledo and Y.L. Tong, A parametrix for $\bar{\partial}$ and Riemann-Roch in Cech theory, Topology 15 (1976), 273-301.

Department of Mathematics, University of Kentucky, Lexington, KY 40506

E-mail address: vgorb@ms.uky.edu

Department of Mathematics, University of Southern California, Los Angeles, CA 90089

E-mail address: fmalikov@mathj.usc.edu

IHES, 35 Route de Chartres, 91440 Bures-Sur-Yvette, FRANCE

E-mail address: vadik@ihes.fr 